\title{
Accessing Information Sources using Ontologies
}

\author{
D. Sun, H. Jung, C. Hwang, H. Kim, S. Park
}

\author{
Dongeun Sun, Hyosook Jung, Chunsik Hwang, Heejin Kim \\ Department of Computer Science Education \\ Korea University,Anam-dong Seongbuk-gu, Seoul, 136-701, Korea \\ E-mail: sunde41@korea.ac.kr, est0718@comedu.korea.ac.kr, vollfeed@korea.ac.kr, prin@korea.ac.kr
}

Seongbin Park

Department of Computer Science Education

Korea University,Anam-dong Seongbuk-gu, Seoul, 136-701, Korea

E-mail: hyperspace@korea.ac.kr

Corresponding author

\begin{abstract}
In this paper, we present a system that helps users access various types of information sources using ontologies. An ontology consists of a set of concepts and their relationships in a domain of interests. The system analyzes an ontology provided by a user so that the user can search and browse Wikipedia [1], DBpedia [4], PubMed [5], and the Web by utilizing the information in the ontology. In particular, terms defined in the ontology are mapped to Wikipedia pages and the navigation history of a user is saved so that it can serve as a personalized ontology. In addition, users can create and edit ontologies using the proposed system. We show that the proposed system can be used in an educational environment.
\end{abstract}

Keywords: Wikipedia, ontology, education.

\section{Introduction}

As the amount of information available from various information sources such as the Web is increasing, it becomes more difficult to find relevant information in large information spaces. In this situation, an ontology that describes concepts and their relationships in a domain of itnerests can help since terms provided by ontologies can help novices within a specific domain or people who are not familiar with searching [22]. An ontology can be also utilized for effective navigation [28].

In this paper, we present an ontology-based system that provides users with appropriate keywords for searching so that users can easily access various types of information sources such as Wikipedia, DBpedia, PubMed, and the Web. The system uses an ontology to provide keywords for searching. If a user selects a concept defined in the ontology, our system shows the corresponding Wikipedia article using http://en.wikipedia.org/wiki/reference, where reference is the selected concept. In addition, the user can access DBpedia dataset and PubMed that contain the information associated with the selected concept. Users can also access the information related to the concept on the Web using Google search engine [21]. On top of these, users can share ontologies with others and the list of pages visited by users can be saved as navigational histories in RDF [6] or OWL [7]. The saved ontology can serve as a personalized ontology that can be utilized for web search personalization [29].

The left figure in Fig. 1 is the screenshot of the page at http://en.wikipedia.org/wiki/Gene which a user can see without using our system. The right figure shows the screenshot of the same web page that a user can see using the proposed system. The ontology viewer shows an ontology imported by a user and the class and individual viewer shows the hierarchy of classes 
in the ontology. When a user clicks a class in the class and individual viewer, the class' detail viewer shows the subclasses and properties of the class. The navigational history viewer shows the list of the pages visited by the user.

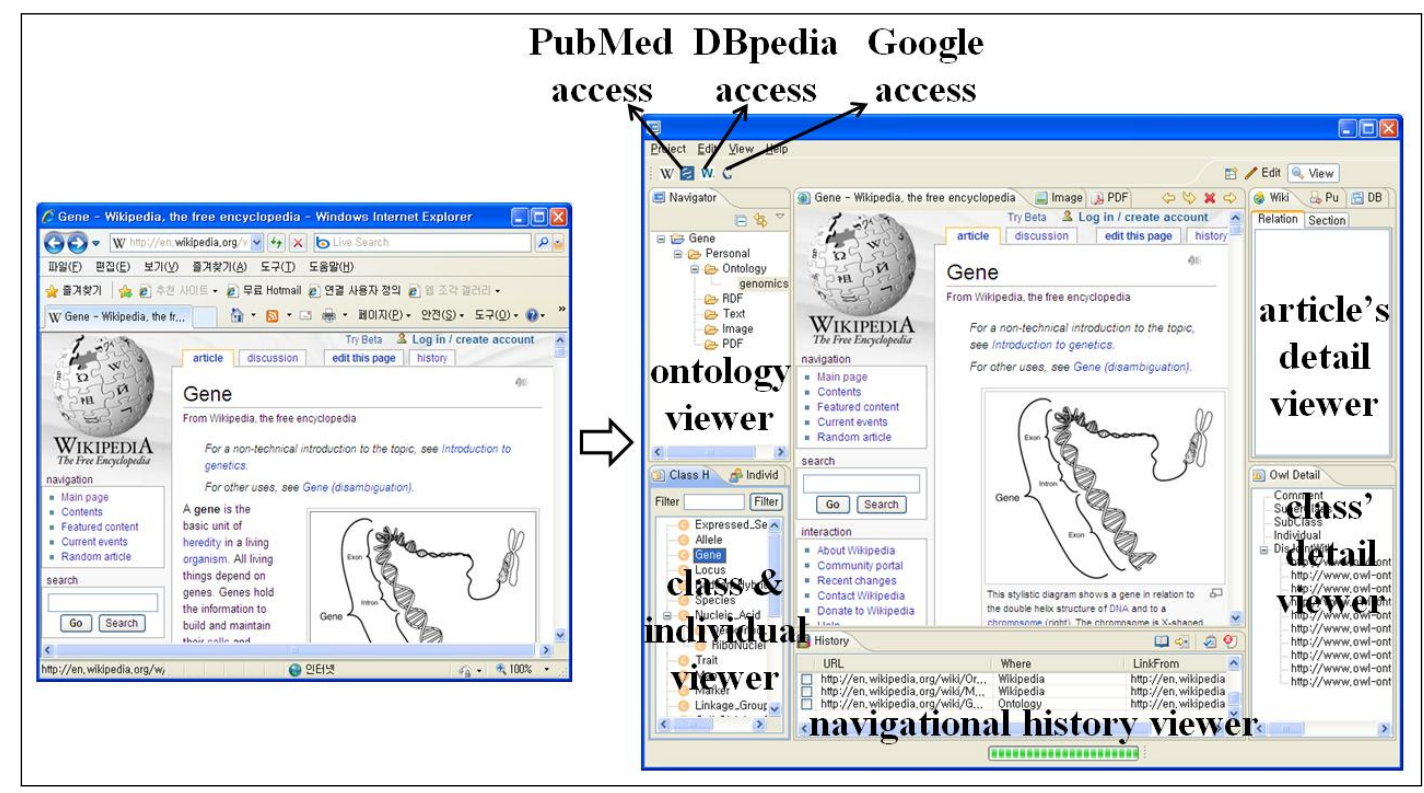

Figure 1: A snapshot of our system

An ontology is usually defined by domain experts and represents essential concepts and meaningful relationships between the concepts. If a user obtains a well-organized ontology defined by domain experts, the user can understand which concepts are suited to the searching goal and which concepts are related meaningfully. It is helpful for the user to establish search strategies. For example, because the user can know which concepts are related to one another, it is easy to determine which concepts the user should look for next. This is important especially in a Web-based educational environment where students who are not sure about how to proceed to find right information for leaning tend to get lost. This symptom is called lost in hyperspace. In such a case, teachers can provide their students with an appropriate navigational guidance by offering ontologies that define important concepts and their relationships so that the students can figure out where to proceed to achieve learning goals by referring to the contents of the ontologies. Ontologies can also help navigating on Web sites since users can click categories defined in ontologies that can be shown on a part of the Web sistes to expand categories.

There are two types of users who can potentially benefit from our system. One is a provider who can create ontologies for consumers. The other is a consumer who uses the ontologies created by the providers. In a learning environment, teachers are providers. Teachers create ontologies by themselves or modify ontologies defined by others using our system. They provide ontologies for their students. Students are consumers. Most probably, they do not know what the ontologies are or how the ontologies are created. They just use the ontologies to search or browse through learning materials. Our system targets on both novices and experts about ontologies. In a more general environment, some users can be both a provider and a consumer. For example, a user might want to define an ontology that captures background knowledge on the domain of interests and utilizes the ontology to improve the accuracy of searching. Depending on the type of a user, our system helps in creating and editing ontologies. For example, an advanced user who knows how to create an ontology can easily edit an existing ontology to personalize it. It is also possible that users can collaboratively edit ontologies. 
This paper is organized as follows. Section 2 describes related works. In section 3 , the functionalities of the system are explained. Section 4 describes illustrative examples that show how the system can be used. Section 5 concludes the paper and describes future works.

\section{Related works}

Ontology is referred to as the explicit and formal specification of a shared conceptualization. As an engineering artifact, it consists of terms and relationships that describe a certain reality, plus a set of explicit assumptions regarding the intended meaning of the vocabulary [8]. An ontology can serve as background knowledge [24] and it can help users refine the search results from domains that they are not familiar with [25]. Constructing a formal ontology generally relies on an interactive process to elicit knowledge and formalize it. The construction of scientific ontologies needs tools required in ontology engineering process because well-formed scientific ontologies can be created when both ontology designers and domain experts collaborate and combine their knowledge [20].

Wikipedia is a freely available online encyclopedia developed by a community of users to which anyone can contribute [1]. Wikipedia supports information collection in e-Science by using Web 2.0 technology. The Gene Wikipedia is a knowledge collection about genes obtained by gathering information from multiple data sources based on a set of ontologies [2]. While Wikipedia is a valuable source of information in many areas, search capabilities are limited to full-text search [3]. [17] sketches a system that helps managing knowledge using Wikipedia. There are browsers that help users access Wikipedia. Pathway is a browser that searches for Wikipedia articles related to a subject and displays the related articles as a graph or network [11]. Gollum allows a user to access Wikipedia through the toolbars and menus and allows the user to add dedicated Wikipedia Bookmarks in a sidebar [12]. Indywiki [13] supports browsing through images by extracting all images on Wikipedia articles related to a search keyword. [18] describes a Wikipedia browsing system using Semantic Web technologies.

Sealife project supports context-based information integration through the use of semantic hyperlinks which map the ontology terms to the web/grid services. It allows users to add the relevant terms to a shopping cart while the users are browsing through various web pages. Then, it presents the contents of the shopping cart and then enables the users to link to the web/grid services according to semantically identified terms [24]. Our system uses a domain specific ontology as background knowledge and links ontology terms to relevant Wikipedia articles. It enables browsing semantically related articles based on the ontology. It also allows users to build a new ontology by using their browsing history that consists of terms automatically extracted from the articles visited by the users. The users can use the ontology in order to browse articles based on user's interest.

Magpie [10] uses an ontology to annotate web pages and provides meaningful information related to the web pages. When a user visits a web page, it annotates semantic entities on a web page based on the ontology. Our system uses an ontology to support the navigation of semantically related web pages within a specific domain. When a user selects a semantic entity, it shows a web page related to the entity and provides supplementary information related to the entity and the web page. In addition, our system allows the users to modify the ontology suited to their needs or interest. It means that it can support the creation and utilization of the personalized ontology.

[15] presents a system that can generate an ontology based on user's seed. A seed is composed of a single sample data instance embedded within the concepts of interest. In our system, a user selects a class in an ontology and the system regards the class as a concept which the user is interested in. The user continues navigating information of interest with following link 
anchors in the article or selecting other classes in the ontology. Our system can create a personalized ontology that consists of information about a history of user's navigation. For example, a class that is selected by a user at first can be considered as a seed and an ontology about the navigational history can be viewed as a generated ontology based on the seed.

[23] presents a system that helps students navigate in the web. It connects each page to one concept of the ontology and creates new links between the pages based on the relationships between the concepts. It can offer semantically related links by using the ontology although there are not real links between the pages. In its experiment, the students who navigated the web pages including semantic links experienced less disorientation, revisited web pages less, spent less time completing tasks, and have done better tasks than those who navigated the general web pages. It means that using ontology can help students quickly and easily find what they want on the web.

[14] is an ontology-based biomedical literature search engine. It retrieves relevant abstracts from PubMed which contain the biomedical concepts related to a search query. The biomedical concepts belong to the Gene Ontology (GO), Medical Subject Headings (MeSH) and Universal Protein Resource (UniProt). In GoPubMed, users can navigate relevant abstracts by selecting one of categorized ontological concepts. GoPubMed supports quick navigation through the abstracts by category and so users can jump to search results related for a certain concept.

Ontoverse [9] creates and links ontological concepts and the information extracted from scientific publications. Ontoverse enables annotating and interlinking knowledge sources and supports cooperative knowledge management within life science based on the ontologies. Our system uses the ontologies as knowledge basis for semantic information access and navigation. Our system obtains ontological concepts from user-defined or existing ontologies and also extracts conceptual information from Wikipedia articles from the history of user's navigation. It is possible to create ontologies collaboratively because it allows users to edit existing ontologies and build new ontologies according to their interest.

When a user navigate in a complex hyperspace, the user can feel the symptom of lost in hyperspace. Our system helps users navigate in a large hyperspace such as the Web using ontologies so that they can find right kind of information easily without being distracted. This is important in an area such as Web-based education, where one type of users is students who need to understand learning materials on the Web. They could have different backgrounds and ontology-based guidance can help them access necessary learning materials. Our system allows teachers to create ontologies suited to their students. It presents the students the ontological concepts related to information to be learned. The students navigate the web according to relationships between the ontological concepts. The system can prevent the students from getting lost in hyperspace or cognitive overload while browsing web pages. In addition, our system can provide adaptation by cooperating with an adaptive hypermedia system. It offers users additional information suited to user's characteristics such as knowledge, interest, etc. In the learning environment, our system can provide students the learning content suited to their learning ability.

Fig. 2 shows how our research is related to other works. Our system uses an ontology to search for semantic information such as Sealife and Magpie and to guide navigation in hyperspace semantically like ontology-based web navigation guidance. It also generates personalized ontology to retrieve information suited to user's need or interest like seed-based ontology generation. Our system enables users to search Wikipedia articles such as Pathway, Gollum and Indywiki and abstracts of biomedical literatures form PubMed like GoPubMed. It also supports searching information from Google and DBpedia. Like Ontoverse, our system supports cooperative ontology building.

The differences between our system and systems mentioned in this section are as follows.

Sealife and Magpie are semantic web browsers to identify ontological terms from web pages 


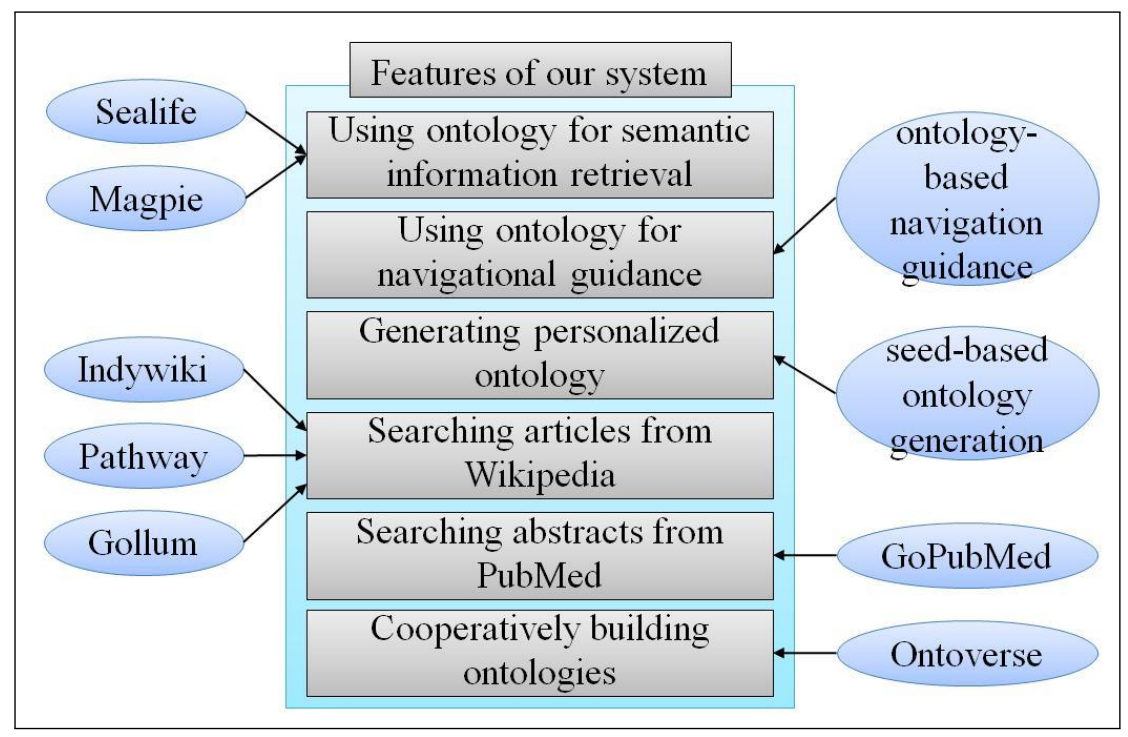

Figure 2: Related works to our research

and enable users navigate the web according to the relationships between the identified terms. The ontology-based navigation guidance lets users navigate web pages which are linked according to the relationships between concepts in ontologies. The seed-based ontology generation and Ontoverse are tools which generate personalized ontology consisting of the concepts of user's interest and gathers information based on the ontology. In order to use these tools, the uses should know ontologies to be used. However, in our system, even though the users like students do not know them, students can search for information by using ontologies defined by experts or teachers.

Pathway, Indywiki, and Gollum are web browsers to support navigating Wikipedia. Pathway keeps track of what users have read on Wikipedia and displays the track in a data map. Indywiki extracts images from related pages on Wikipedia and displays the page that the image links to. Gollum gives users special functions which support working with Wikipedia easily. When using these tools, the use should choose proper queries to find for information. However, our system helps the users to determine the queries as showing the concepts related to the information to be searched.

GoPubMed is a search engine to search for abstract of biomedical literatures from PubMed, $\mathrm{GO}, \mathrm{MeSH}$, and UniPort. While it is too professional for novices about biology to retrieve proper information, out system helps the users search for information about different domains as well as biology by using ontologies customized according to the user's features.

\section{Functionalities of the system}

In this section, we explain the functionalities provided by our system. The system parses an ontology and shows the classes and individuals of the ontology in a tree structure. A user can use one's own ontology or an ontology found from Swoogle which is a Semantic Web search engine [16]. Users can add new classes or individuals to the ontology or remove the classes or individuals from the ontology. If a user selects a class, it shows the subclasses, properties, or individual of the class in a tree structure. The system also displays the corresponding Wikipedia article. In addition, it provides concept categories which the article belongs to and incoming links to the article. 
Fig. 3 depicts the functionalities of the proposed system.

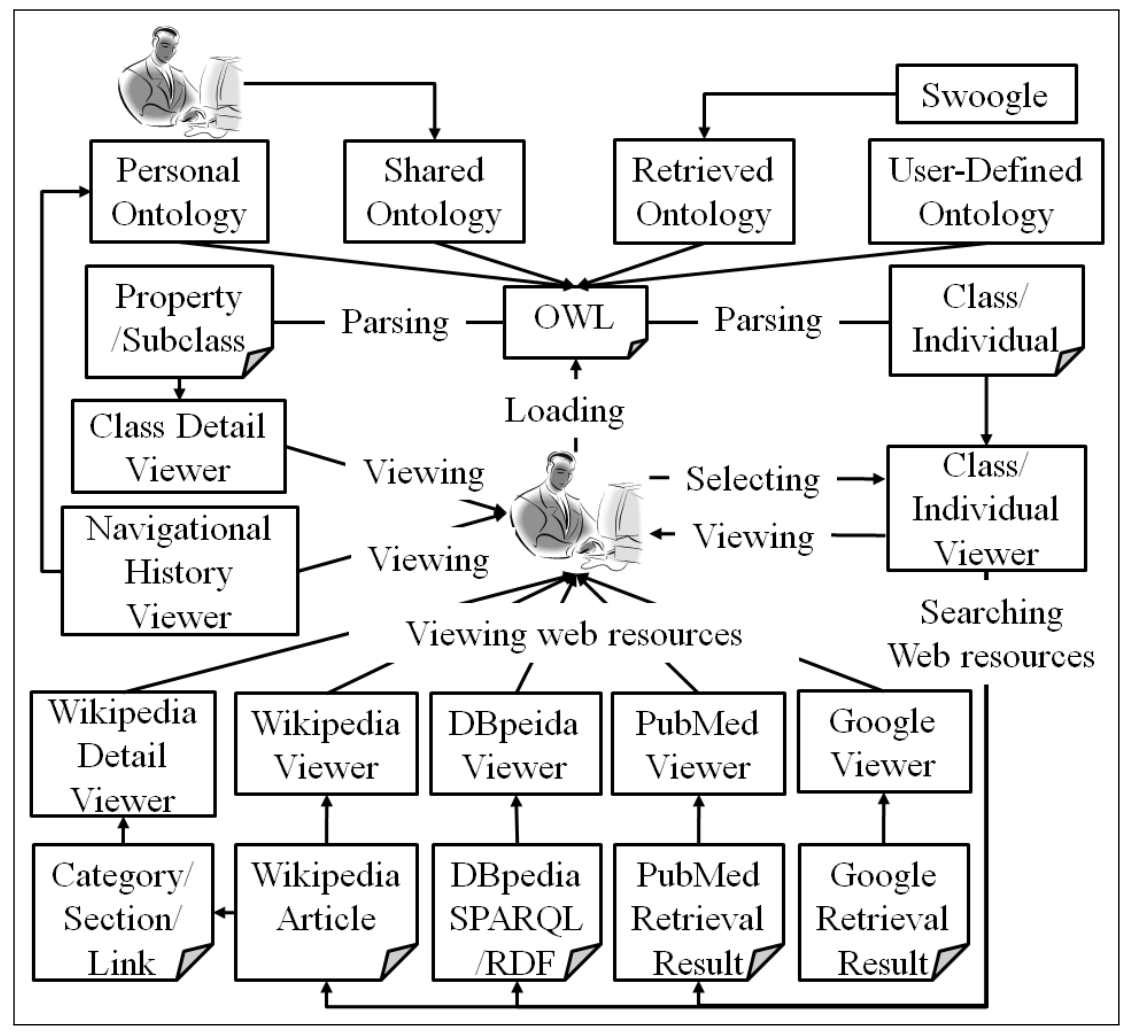

Figure 3: Functionalities of the proposed system

A user can save text data, image, PDF files into project, which is the data collection including ontology, text, image, PDF in our system. Besides, for saved data in project, the system has its own viewing function of TEXT, image (jpg, gif, PNG format), PDF. The system supports OWL editing such as syntax coloring, formatting, highlighting, content outline, word wrap and so on. A user can export information about the Wikipedia articles visited by the user in an ontology. The representative concept of accessed articles is automatically created as individuals of the ontology.

\subsection{Ontology-based browsing}

A user can retrieve information from DBpedia by selecting a class and clicking a DBpedia button. Fig. 4 shows an RDF document corresponding to the selected class from DBpedia.

When double-clicking a class 'protein' in DBpedia viewer, the user can see the HTML page which the RDF document of 'protein' is transformed into. If clicking an icon on the bottom of the page, the user can visit a web page to use SPARQL query for searching DBpedia dataset. If the user types a query in the text box like "select distinct? Protein where [] a ?Protein" and clicks a button 'Run Query', the user can see information related to the query such as data related to 'protein' (Fig. 5)

It is also possible that the user can access information from PubMed which is an archive of biomedical and life sciences journals. It provides a list of the publications related to a selected class or individual and shows information about a selected publication such as authors, abstract, title, etc. 


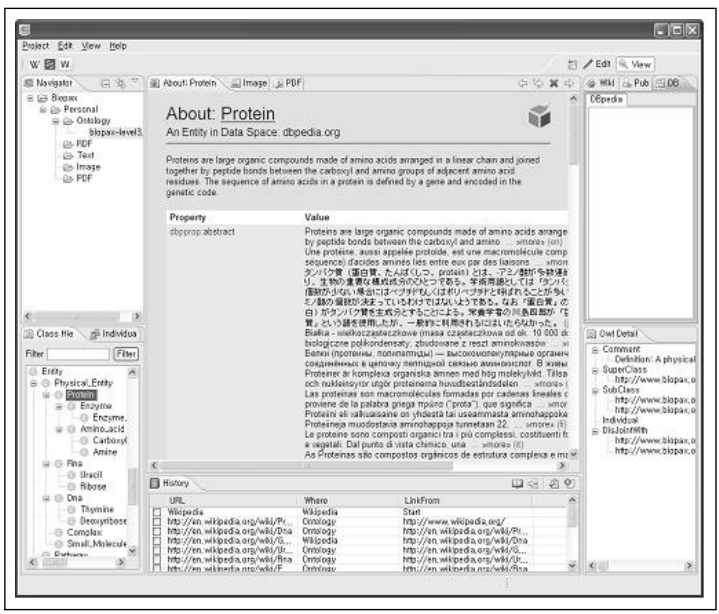

Figure 4: A result from DBpedia

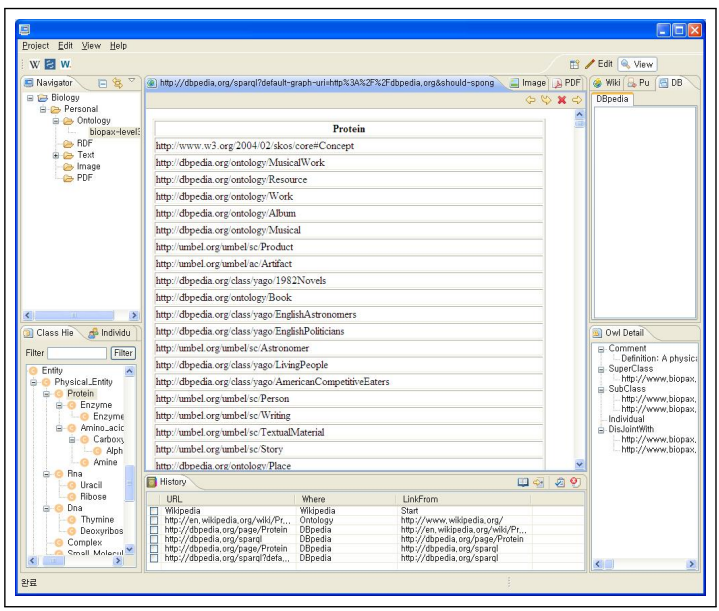

Figure 5: SPARQL query result 


\subsection{Collaborative ontology building}

Users can write an ontology collaboratively with other users while sharing their opinions and knowledge for their common goal. For example, an expert accesses a Wikipedia article about a class Protein. While reading the article, he wants to know information about Amino acid. He continues navigating the article about Amino acid. Then, he edits the ontology by adding a subclass Amino_acid of the class Protein. He also adds subclasses Carboxyl_group and Anime of the class Amino_acid. He shares the ontology with other experts. (Fig. 6)

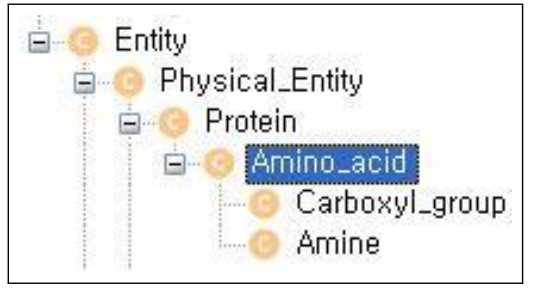

Figure 6: Editing a part of a shared ontology

Now, another expert interested in Enzyme downloads the edited ontology. She follows a link anchor Enzyme while reading the Wikipedia article about Protein and continues reading the article about Enzyme. Then, she edits the ontology by adding a subclass Enzyme of the class Protein and a subclass Enzyme_catalysis of the class Enzyme. (Fig. 7)

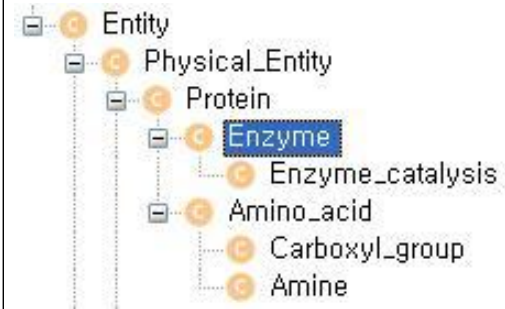

Figure 7: Editing parts of the shared ontology

\subsection{Creation of a navigation history}

Our system exports the navigation history that consists of the information about pages visited by a user in OWL and it can serve as a personalized ontology which can be used for semantic searching. For example, a personalized ontology can be created as follows. (Fig. 8)

1. A user is interested in water activities and loads an ontology about travel (travel.owl).

2. The user clicks individual kayaking of class Water_activity for requesting a Wikipedia article about kayaking.

3. To return the Wikipedia article corresponding to the class, the system creates a URL string including a class name as a query like http://en.wikipedia.org/wiki/Name, where Name is a concept or term to search for the article. Our system creates a URL string including the individual name like "http://en.wikipedia.org/wiki/Kayaking".

4. Wikipedia sends the article Kayaking and our system shows the article to the user. 
5. While navigating in Wikipedia, the user is interested in canoeing which is similar kayaking. The user adds canoeing as a new individual of class Water_activity to the ontology.

6. The user can see the article Canoeing by clicking the individual canoeing.

7. The user can obtain RDF data of DBpedia about canoeing.

8. The user can also obtain searching results of PubMed about canoeing.

9. The user continues navigating other interesting Wikipedia articles such as Niagara_Falls, Horseshoe_Falls, Whitewater_canoeing, etc. Then, the user builds an ontology which consists of the navigational history of the user. In the ontology, the visited articles are represented as individuals of class Article.

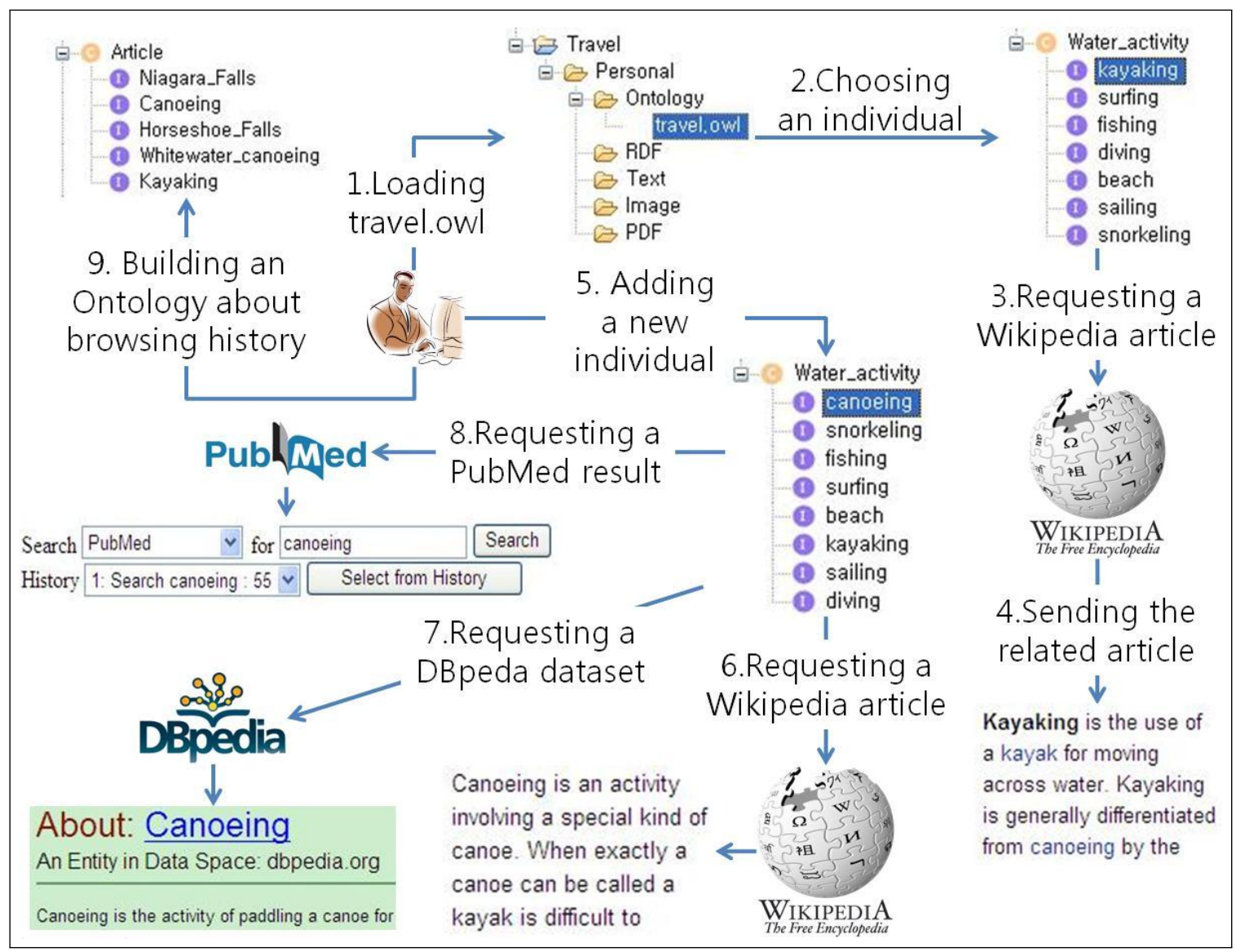

Figure 8: Creation of a personalized ontology

Many ontologies are defined by domain experts. While one important reason to use ontologies is to support knowledge sharing for different types of applications, ontologies can be also re-used and customized for personal use. Especially, since the number of ontologies on the Web is everincreasing and there are search engines for ontologies, average users can find ontologies for the domain of interests under consideration easily. If the users can edit ontologies found on the Web using either our system or other ontology editors, they can customize them so that the modified ontologies can be used for interesting applications. One area where this can help is Web-based education where teachers can prepare a set of concepts or terminologies that need to be accessed by students on the Web. In this situation, teachers serve as so-called trail blazers who provide 
helpful information for someone else. It is also possible that personalized ontologies can help learning in adaptive hypermedia area since different types of adaptations can be made possible using the information contained personalized ontologies. In other words, a base ontology exists and there also exist different ontologies that are modified slightly from the base ontology in order to support adaptive learning.

\subsection{An illustrative example}

In this section, we show navigational efficiency of our system by comparing navigation using our system and navigation using a general web browser.

We believe that users can access information from different websites faster by using our system than by visiting each web site manually. In our system, they can search information from different websites in an application. They do not need to load web pages in a web browser, type the URLs of each web site, and enter queries. Instead, they can navigate the web sites by clicking terms defined in ontologies. As an illustrative example, let's assume that a teacher wants students to find out information about human nutrition source in Wikipdia. Specifically, the students should find out Wikipedia articles about "Photosynthesis", "Herbivore", and "Carnivore" but the problem is that students do not know the terms exactly. So, a typical way of finding the information using a web browser would be that a student first visits Wikipedia and types query "nutrition" in the search box. The student reads the Nutrition article and clicks link anchor "food" which is shown in the left figure in figure 9. Then the student reads the Food article and clicks "plant" which is shown in the right figure in figure 9.

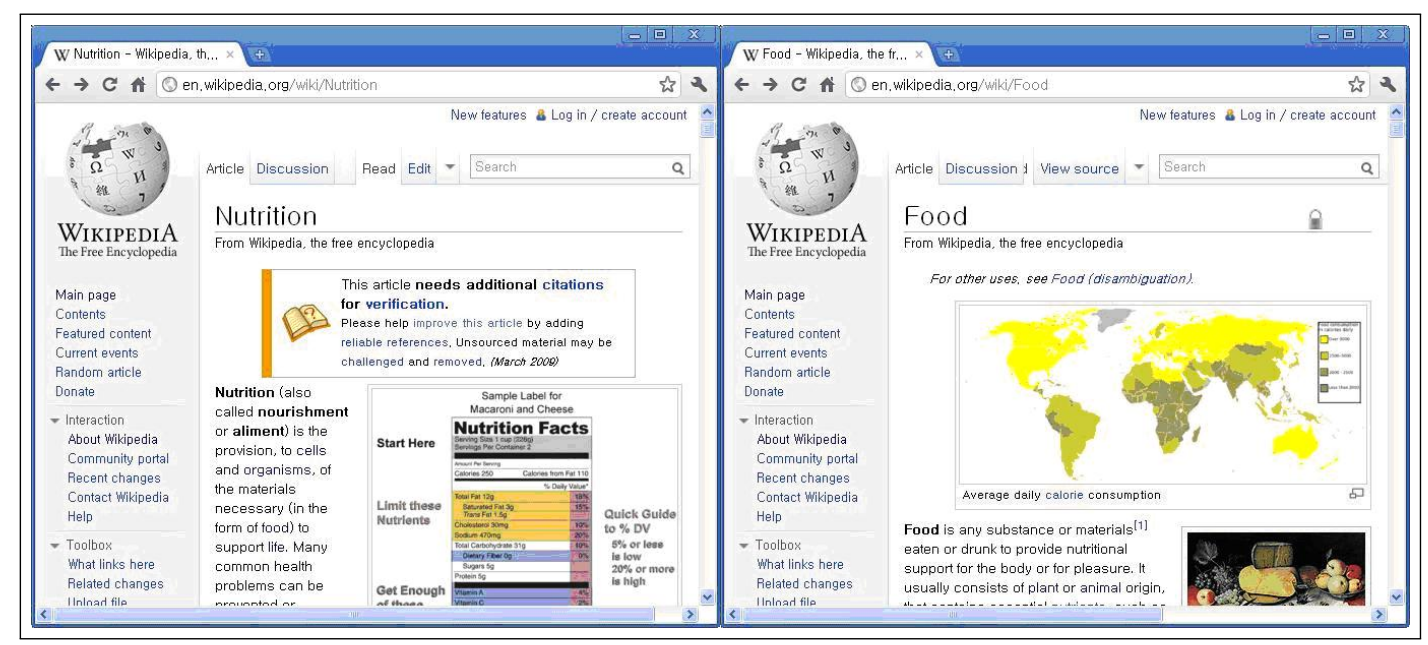

Figure 9: Search results using a general web browser

After that, the student reads the Plant article and clicks "photosynthesis" and eventually finds the Photosynthesis article that is shown in the left figure in figure 10. Then, the student goes back to the Food article and clicks "animal" which is shown in the right figure in figure 10.

Now, the student reads the animal article and clicks "carnivore" and "herbivore". Then, student eventually finds the Herbivore article and the Carnivore article as shown left figure in figure 11 and right figure in figure 11, respectively.

In our system, a student can open an ontology defined by the teacher. The student clicks "Photosynthesis" on the class view and then obtains the Photosynthesis article immediately which is shown in figure 12 .

Then, the student also clicks "Herbivore", and "Carnivore" on the class view and then obtains the Herbivore article and the Carnivore article which is shown in left figure in figure 13 and right 


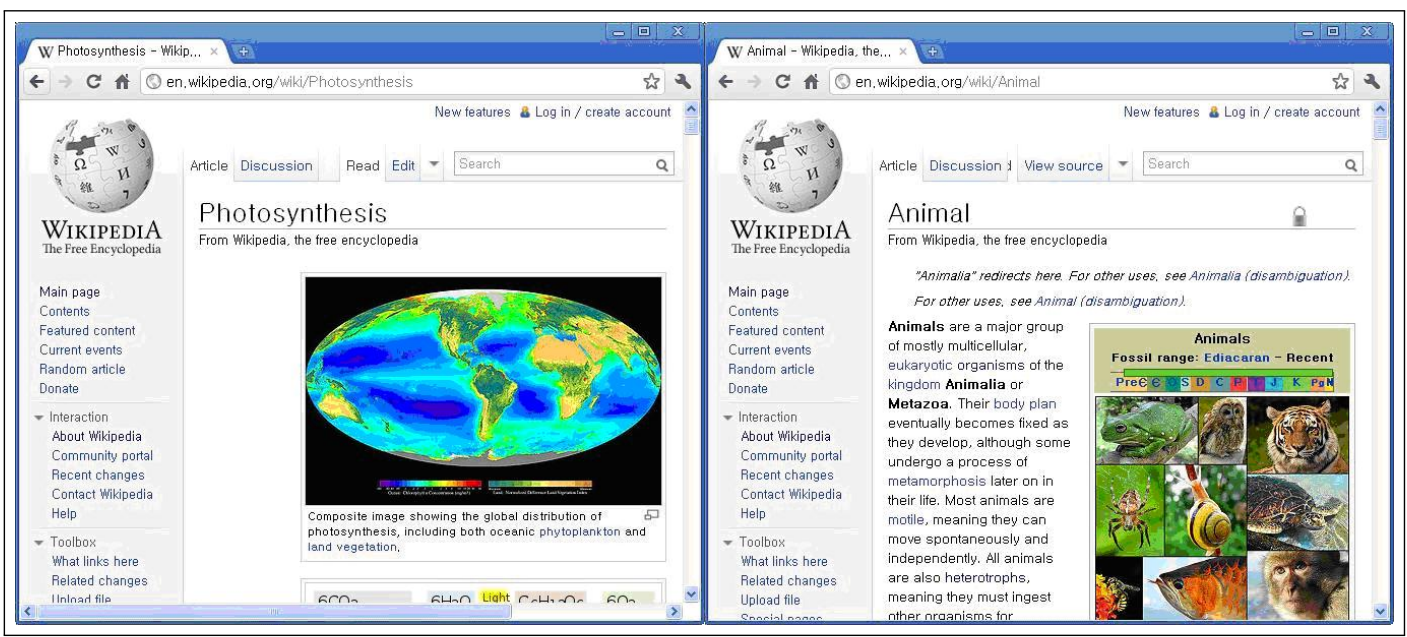

Figure 10: Search results using a general web browser

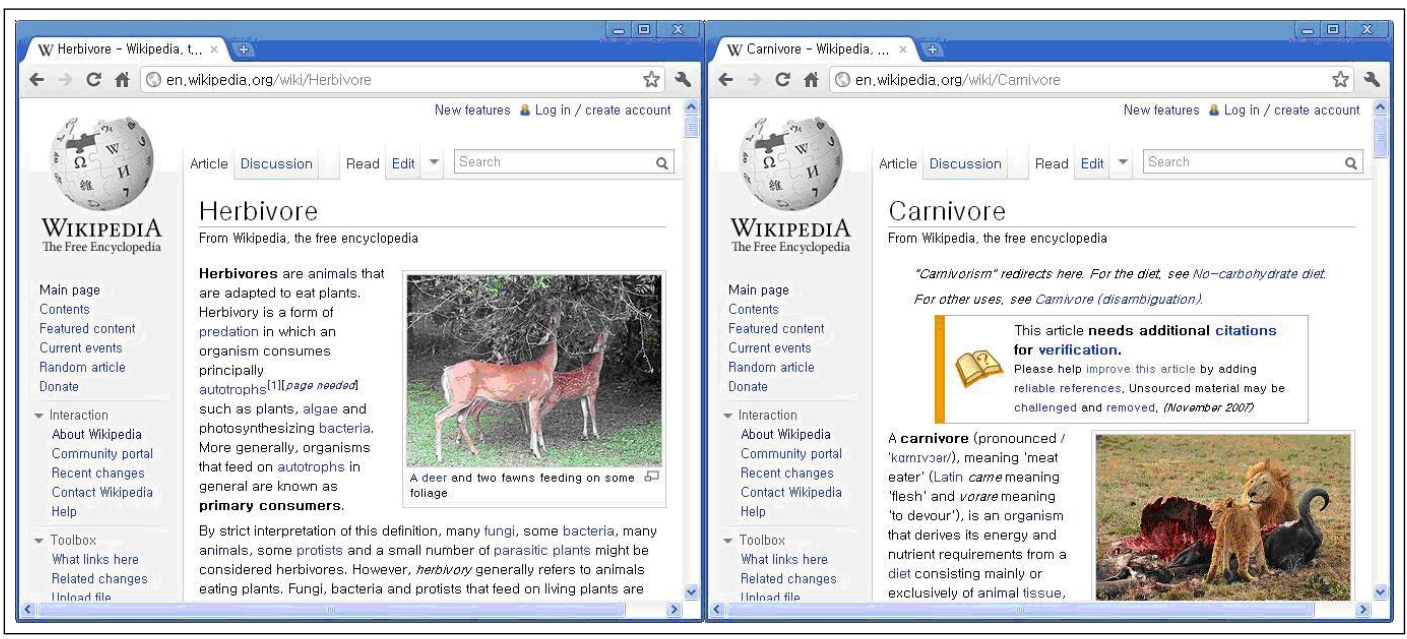

Figure 11: Search results using a general web browser

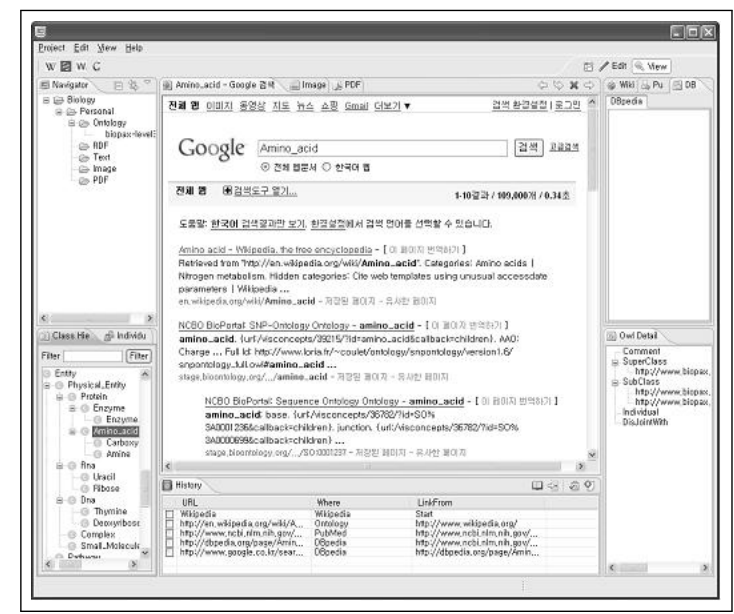

Figure 12: Screenshot of our system 
figure in figure 13, respectively.

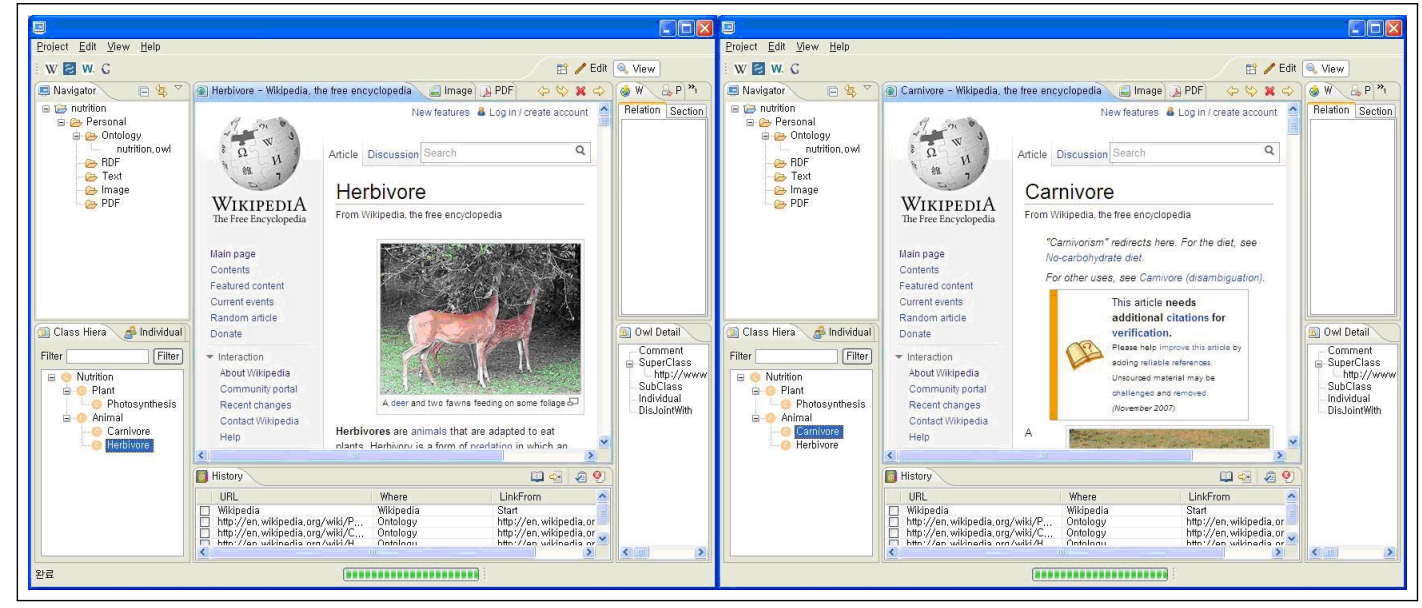

Figure 13: Search results using our system

\section{Applications}

In this section, we show how the proposed system can be used in an educational environment.

\subsection{Management of studying materials on the Web}

Assume that a student is studying the history of the Middle Ages by browsing resources on the web. The student wants to organize information by concepts related to events or developments of the middle ages. Our system can help the student manage the information conveniently.

Fig. 14 is the screenshot of the system, where the student loads an ontology related to the history of the Middle Ages and their relationships. For example, it defines classes such as war, health, religion, building, etc. It defines subclasses of each class like the subclasses of class building are castle, dungeon, walled town, moat, etc. If the student double clicks a class, the student can see a Wikipedia article corresponding to the class. The student reads the article and saves a part of the article inside the system.

If the student wants to search health information related to the Middle Ages such as the Bubonic plague and herbal cure, the student selects a class the bubonic plague and clicks an icon "PubMed". The student can see a list of the publications retrieved from PubMed. (Fig. 15)

The student can also search the RDF documents related to the Wikipedia articles from DBpedia. (Left figure in Fig. 16) In addition, the student can see a list of retrieval results from Google. (Right figure in Fig. 16)

The navigational history is recorded while the student browses web pages. Like bookmarks, the student can save the information about visited web pages in OWL or RDF which can serve as a personalized ontology.

\subsection{Utilization of a navigation history}

Assume that a teacher plans to teach about cell. In the class, the teacher is planning to make the students search useful resources related to the lesson in the web after teaching basic concepts. However, it is hard for them to search or define appropriate ontologies. So, the teacher 


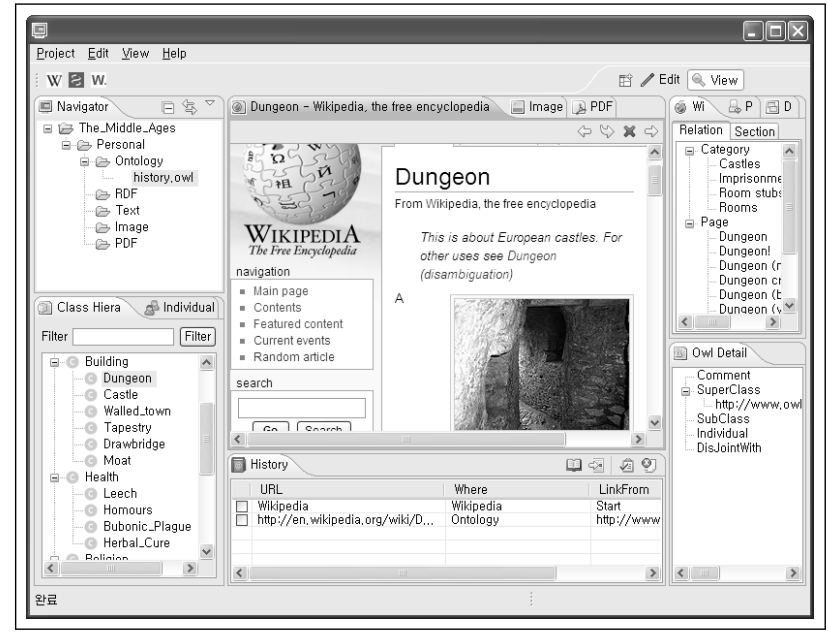

Figure 14: Initial screenshot

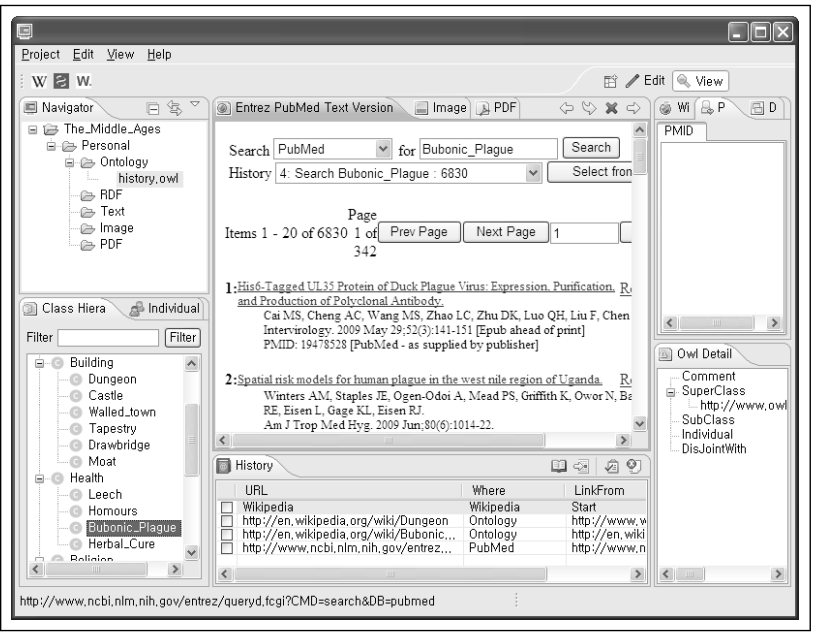

Figure 15: A result from PubMed

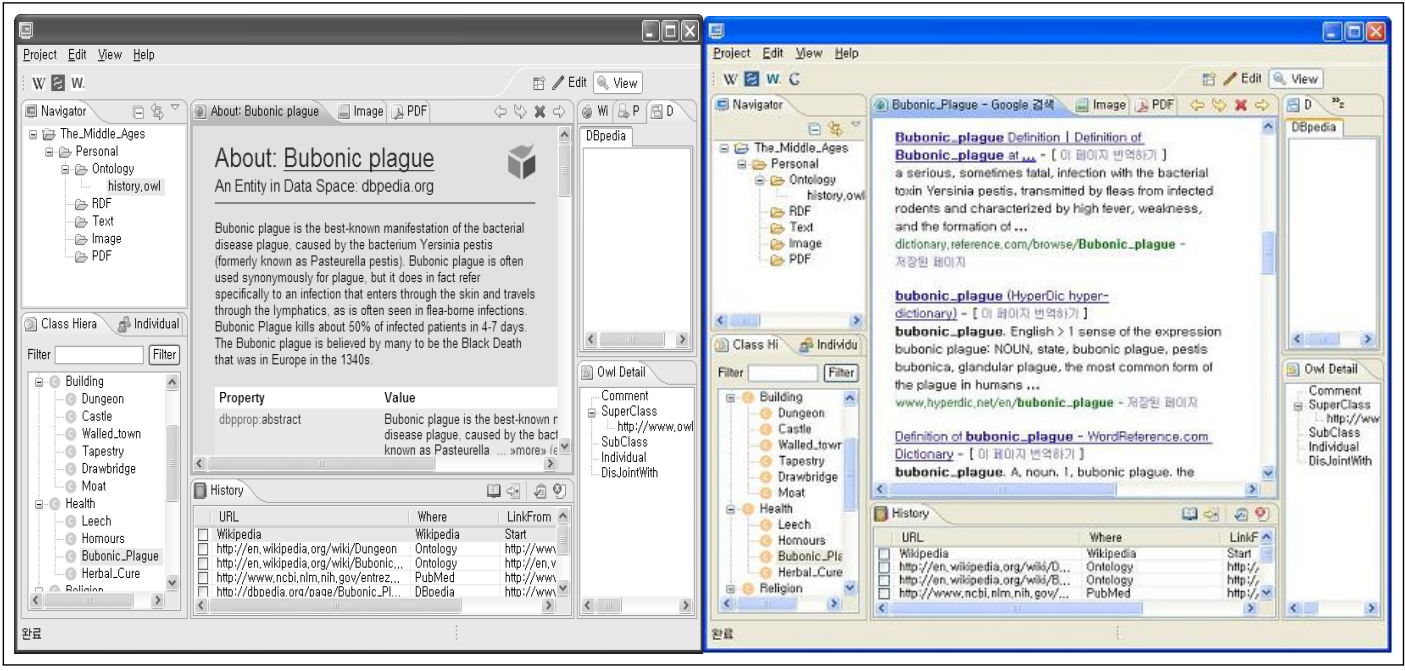

Figure 16: A result from DBpedia (left figure) and a result from Google (right figure) 
needs to provide the students with an ontology which contains basic concepts. Using our system, the teacher browses Wikipedia articles and occasionally the teacher selects a certain class of the ontology and browses its Wikipedia article. When reading the article, the teacher can find essential concepts even though they are not defined in the basic ontology yet. By clicking a certain word in the article, the teacher can browse the Wikipedia articles related to the word. The teacher can save the navigational history as an OWL document where the articles are saved as the individuals of Article class. For example, when reading Mitosis article, the teacher also reads articles about DNA, RNA and virus. Although the basic ontology does not contain the concepts, the teacher wants to make the students read the articles. So the teacher saves navigational history about DNA, RNA and virus article. Using the system, the students can load the ontology of navigational history where DNA, RNA and virus are saved as the individuals of Article class when the teacher saves the navigational history. They select a certain class of the ontology and request information about the concept from Wikipedia, Google, PubMed and DBpedia. A student selects Virus class and asks virus article from Wikipedia. (Left figure in Fig. 17) The student wants to find out news, blogs, and images related to virus. So, the student asks information from Google after selecting Virus class. (Right figure in Fig. 17)

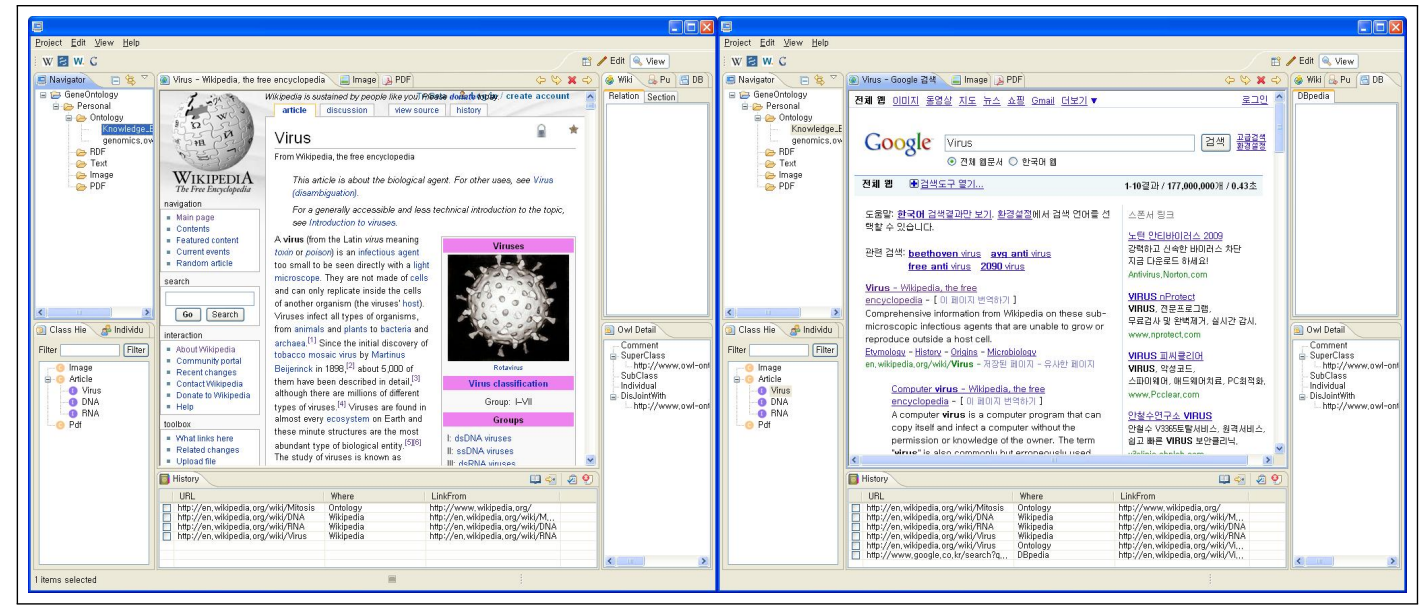

Figure 17: Wikipedia article (left figure) and Google searching results (right figure)

If the student wants to find journals related to virus, the student can request PubMed to search journals after selecting Virus class using our system. The student can also obtain structured data of Wikipedia article (i.e., an RDF document) about virus from DBpedia. On top of these, students can find semantically-related document to the ontology on the web using a semantic search engine [19] where the ontology can serve as searching context. Fig. 18 is the screenshot of the semantic search engine, where individuals of the loaded ontology are shown. The semantic search engine extracts terms from the ontology that are its individual names such as DNA, RNA, Mitochondria, and Ribosome. They are used as keywords for searching. For example, [19] can generate a query string from these keywords and find Web documents or Semantic Web documents using the query string.

\subsection{Interacting with an adaptive hypermedia system}

Our system can interact with AHA! which is an adaptive hypermedia system [27] that has been developed in order to offer links and content suitable to each user so that the user cannot be disoriented during navigation in a hyperspace. AHA! server consists of domain model, user model and adaptation model. Domain model describes the structure of domain and user model 


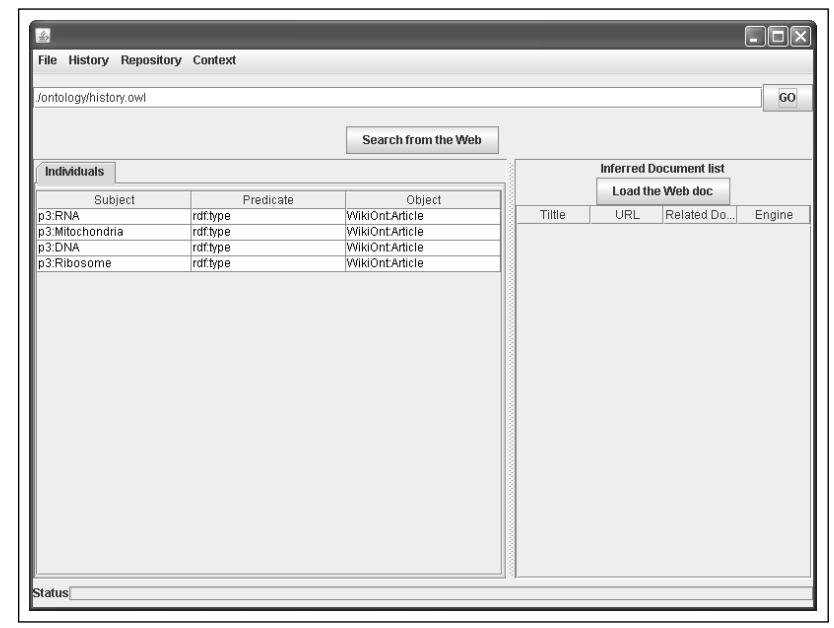

Figure 18: Individual information

contains the information about users. Adaptation model describes how adaptation can be made depending on a situation. Our system can use an ontology that consists of concepts of the domain model. The classes of the ontology are the concepts of the domain model and the hierarchy of the classes is the same as that of the concepts. When a user accesses a web page in AHA! server, the user can also access additional information related to the web page which is automatically provided by our system.

For example, an author creates a tutorial about markup language in AHA! server. The domain model consists of concepts about markup language such as HTML, XHTML, XML, etc. Each concept has a corresponding web page. In our system, there is an ontology that consists of classes corresponding to the concepts. A user is accessing a web page about xhtml in AHA! server that is one of the concepts in domain model. (Fig. 19)

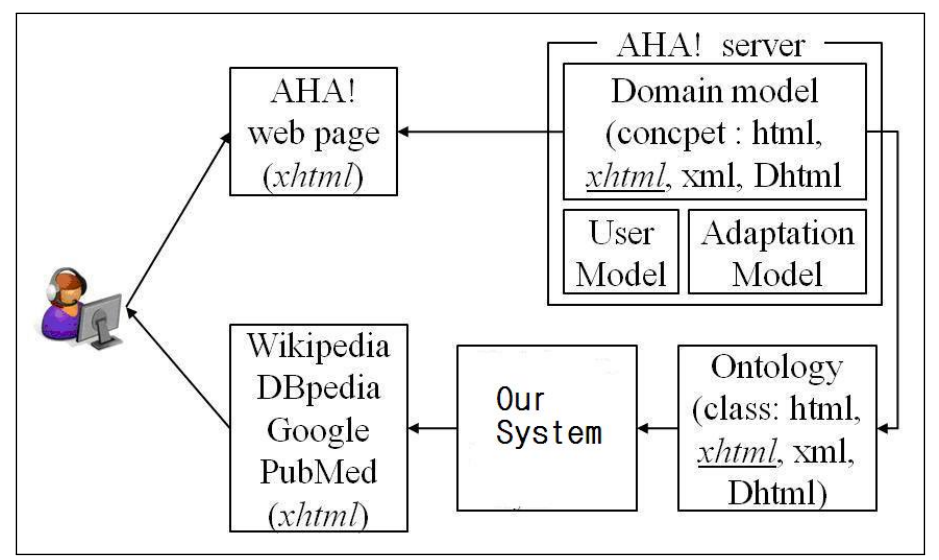

Figure 19: Interaction between our system and AHA! system

The user wants to read additional information related to xhtml. So, the user clicks "additional information" on the top of the web page that is available from the AHA! server. Clicking "additional information" makes our system show a Wikipedia article about XHTML by loading the ontology that consists of the concepts of the current domain model and receiving the concept xhtml which the user is accessing to. (Fig. 20)

Similarly, the user can also access the information from DBpeida, PubMed, and Google. 


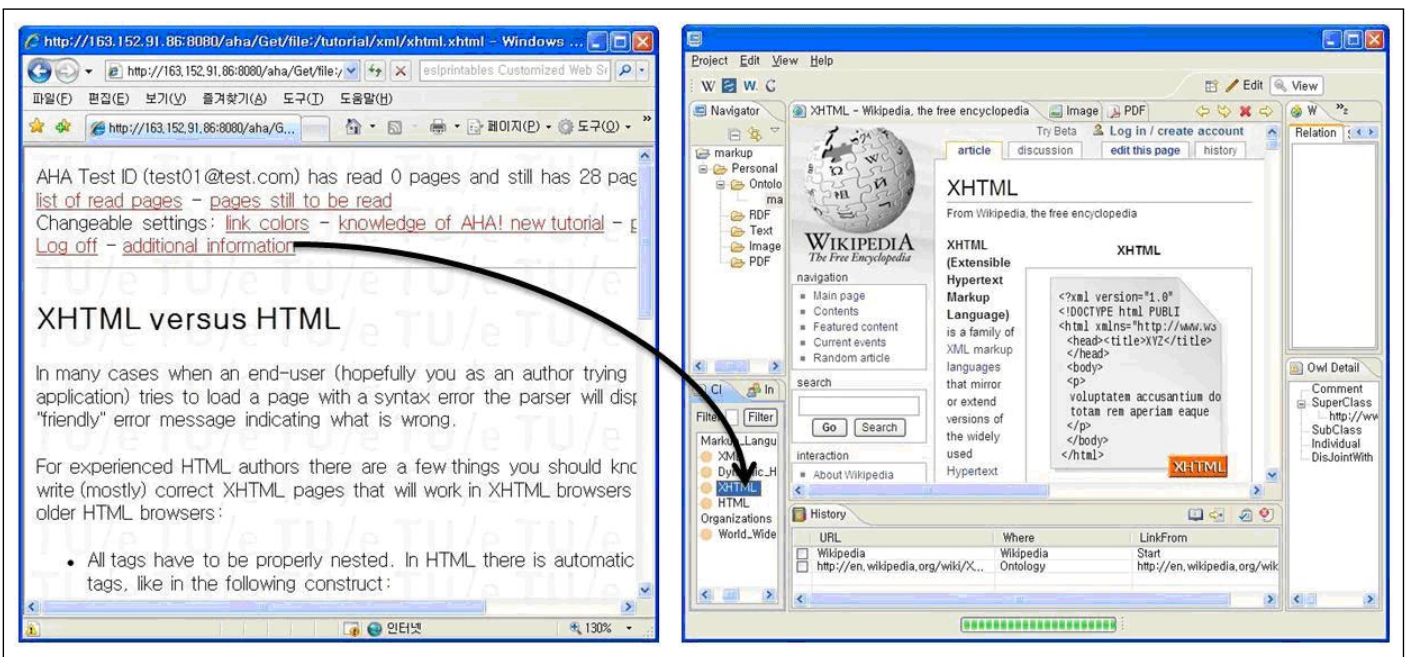

Figure 20: Accessing additional information from Wikipedia

\section{Conclusion and Future works}

In this paper, we presented an ontology-based system that can help users access various types of information sources. Using our system, users can browse Wikipedia articles, Google searching results, PubMed journals, and DBpedia data about a certain concept defined in an ontology in a single user interface. They can also find out other concepts semantically related to the concept by traversing ontological hierarchies between the concepts. Our system provides an environment for collaborative ontology engineering that enables users to create, edit and maintain ontologies and to reuse the ontologies created by other users. The users build new ontology projects and open ontologies created by others and edit them. In addition, they store information about articles navigated on the Wikipedia and ontologies are automatically created.

The contributions of the paper are as follows. First, the proposed system supports the integrated and uniform way of searching and browsing information contained in different data sources using ontologies. Second, users can create and maintain personalized navigation histories that can be utilized for searching. Third, the system allows users to build ontologies collaboratively.

We are currently working on ways by which information about metadata and ontologies can be utilized in our system as [26].

\section{Bibliography}

[1] Wikipedia, 2009. Available from http://en.wikipedia.org/wiki/WikiPedia.

[2] Gene Wikipedia, 2006. Available from http://www.bioinformatics.org/genewiki/wiki.

[3] Auer, S., Bizer, C., Kobilarov, G., Lehmann, J., Cyganiak, R., Ives, Z., 2007. DBpedia: A Nucleus for a Web of Open Data. Proc. 6th Int. Semantic Web Conf. and 2nd Asian Semantic Web Conf. (ISWC+ASWC 2007), Busan, Korea. p.722-735.

[4] DBpedia, 2009. Available from http://dbpedia.org.

[5] PubMed, 2009. Available from http://www.ncbi.nlm.nih.gov/pubmed.

[6] RDF, 2004. Available from http://www.w3.org/RDF. 
[7] OWL, 2004. Available from http://www.w3.org/TR/owl-ref.

[8] Ontology, 2002. Available from http://km.aifb.uni-karlsruhe.de/projects/owl/index.html

[9] Ontoverse, 2005. Available from http://www.ontoverse.org.

[10] Magpie, 2005. Available from http://projects.kmi.open.ac.uk/magpie/main.html.

[11] Pathway, 2007. Available from http://pathway.screenager.be.

[12] Gollum, 2009. Available from http://gollum.easycp.de/en.

[13] Indywiki, 2008. Available from http://indywiki.sourceforge.net.

[14] GoPubMed, 2009. Available from http://www.gopubmed.com.

[15] Tao, G., Embley, D. W., 2007. Seed-Based Generation of Personalized Bio-ontologies for Information Extraction. In Proceedings of the Advances in Conceptual Modeling - Foundations and Applications, p.74-84.

[16] Swoogle,2007. Available from http://swoogle.umbc.edu.

[17] Sun, D., Park, S., Jung, H., 2009. Knowledge management using Wikipedia. Semantic Web Applications and Tools for Life Sciences, Proc. CEUR, Vol. 435, Edinburgh, United Kingdom.

[18] Sun, D., 2009. Design and Implementation of a Wikipedia Browser using Semantic Web Technologies, master's thesis, Korea University, Korea.

[19] Ahn, J., Jung, H., Kim, H., Sun, D., Park, S., 2008. A system for contextual search. Proc. IEEE Int. Workshop on Semantic Computing and Applications, p.96-98.

[20] Mainz, I., Weller, K., Paulsen, I., Mainz, D., Kohl, J., von Haeseler, A., 2008. Ontoverse: Collaborative Ontology Engineering for the Life Sciences. Informations-Wissenschaft und Praxis, Vol. 2, p.91-99.

[21] Google, 2009. Available from http://www.google.com.

[22] Groth, K., Lannerö, P., 2006. Context browser: ontology based navigation in information spaces. Proc. 1st Int. Conf. on information interaction in Context, IIiX: Vol. 176, p.75-78.

[23] Jung, H., Kim, H., Min, K., Park, S., 2009. The Ontology-based Web Navigation Guidance System. The Journal of Korean Association of Computer Education, Vol. 12(5).

[24] Schroeder, M., Burger, A., Kostkova, P., Stevens, R., Habermann, B., Dieng-Kunts, R., 2006. Sealife: A Semantic Grid Browser for the Life Sciences Applied to the Study of Infectious Diseases. Vol. 120, p.167-178.

[25] Bonomi, A., Mosca, A., Palmonari, M., Vizzari, G., 2008. Integrating a Wiki in an Ontology Driven Web Site: Approach, Architecture and Application in the Archaeological Domain. 3rd Semantic Wiki Workshop.

[26] Bhagdev, R., Chapman, S., Ciravegna, F., Lanfranchi, V., Petrelli, D., 2008. Hybrid Search: Effectively Combining Keywords and Semantic Searches. Springer Berlin / Heidelberg, p.554568.

[27] De Bra, P., Brusilovsky, P., Houben, G., 1999. Adaptive Hypermedia: From Systems to Framework. ACM Computing Surveys, Vol. 31(4). 
[28] Velart, Z., Saloun, P., 2007. Ontology Based Course Navigation. Proc. 18th Conf. on Hypertext and Hypermedia, p.151 - 152.

[29] Sendhilkumar, S., Geetha, T. V., 2008. Personalized Ontology for Web Search Personalization. Annual Bangalore Compute Conference. Proc. 1st Bangalore Annual Compute Conf., p.1-7. 